\title{
Contents of Volume 139 (2003)
}

Volume 139 No. 1, October 2003

Mark Goresky and Yung Sheng Tai: The Moduli Space of Real Abelian Varieties with Level Structure

Ali Baklouti and Hidénori Fujiwara: Opérateurs différentiels associés à certaines représentations unitaires d'un groupe de Lie résoluble exponentiel

Bogdan Ion: Involutions of Double Affine Hecke Algebras

Douglas Bowman and David M. Bradley: Resolution of Some Open Problems Concerning Multiple Zeta Evaluations of Arbitrary Depth

Margherita Roggero and Paolo Valabrega: The Speciality Lemma, Rank 2 Bundles and Gherardelli-type Theorems for Surfaces in $\mathbf{P}^{4}$

W. Śliwa: On Fredholm Operators Between Non-archimedean Fréchet Spaces

Volume 139 No. 2, October 2003

Richard Hain and Makoto Matsumoto: Weighted Completion of Galois Groups and Galois Actions on the Fundamental Group of $\mathbb{P}^{1}-\{0,1, \infty\}$

Scott Nollet and Enrico Schlesinger: Hilbert Schemes of Degree Four Curves

Alexandru Buium: Differential Modular Forms on Shimura Curves, I

Volume 139 No. 3, December 2003

Nikolaos Tziolas: Terminal 3-Fold Divisorial Contractions of a Surface to a Curve I

Roberto M. Avanzi and Umberto M. Zannier: The Equation $f(X)=f(Y)$ in Rational Functions $X=X(t), Y=Y(t)$ 
Adrian Diaconu, Dorian Goldfeld and Jeffrey Hoffstein: Multiple Dirichlet Series and Moments of Zeta and $L$-Functions

Wolmer V. Vasconcelos: Multiplicities and Reduction Numbers $361-379$

Author Index, Volume 139 (2003) 